\title{
Electrical and magnetic properties of $\mathrm{Si}$ ion implanted $\mathrm{YBa}_{2} \mathrm{Cu}_{3} \mathrm{O}_{7-\delta}$ thin films and microbridges
}

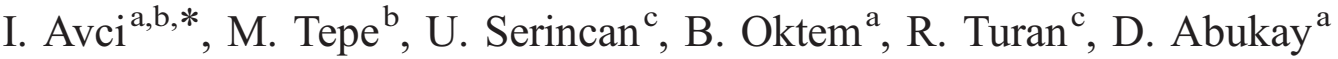 \\ ${ }^{a}$ Izmir Institute of Technology, Department of Physics, Urla Campus, 35437 Urla-Izmir, Turkey \\ ${ }^{\mathrm{b}}$ Ege University, Department of Physics, 35100 Bornova-Izmir, Turkey \\ ${ }^{\mathrm{c}}$ Middle East Technical University, Department of Physics, 06531 Ankara, Turkey
}

Received 3 June 2003; received in revised form 27 January 2004; accepted 27 January 2004

Available online 12 April 2004

\begin{abstract}
Fabrication of superconducting bilayer $\mathrm{YBa}_{2} \mathrm{Cu}_{3} \mathrm{O}_{7-\delta}(\mathrm{YBCO})$ thin film structure by Si ion implantation and properties of microbridge

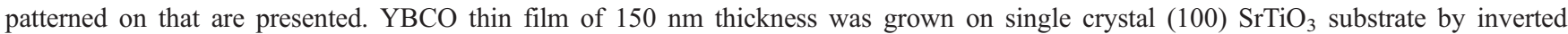
cylindrical magnetron sputtering. The sample was implanted with $100 \mathrm{keV}, 1 \times 10^{16} \mathrm{Si}$ ions $/ \mathrm{cm}^{2}$. Upon implantation with Si, the sample lost its electrical conductivity and diamagnetism while its crystalline structure was preserved after the annealing of the sample. The implanted ions do not alter the overall crystal structure of high temperature superconductor film. This allows the growth of epitaxial superconducting second layer YBCO film on top of the implanted area without using any buffer layer, thus providing an effective method of fabricating multilayer structures. The second layer film and the microbridge patterned by laser writing technique, showed the superconducting properties similar to those of pure YBCO base layer with a reduced critical current density.
\end{abstract}

(C) 2004 Elsevier B.V. All rights reserved.

PACS: 74.76; 74.72.B; 61.72.T

Keywords: $\mathrm{YBa}_{2} \mathrm{Cu}_{3} \mathrm{O}_{7-\delta}$ thin films; Ion implantation; Multilayers

\section{Introduction}

The Josephson junctions are the basic structure elements of the devices used in superconducting electronics and the superconducting quantum interference devices (SQUID). There have been great amounts of research studies on fabrication of superconducting devices using high- $T_{\mathrm{c}}$ oxide superconducting thin films. The most widely used material is $\mathrm{YBa}_{2} \mathrm{Cu}_{3} \mathrm{O}_{7-\delta}(\mathrm{YBCO})$ thin films, which are deposited in monolayer forms on various substrates. Fabrication of devices constructed vertically on a substrate with multilayer processing is highly desirable. This, however, presents a challenge to be overcome due to interlayer diffusion problem that does not allow the layers electrically be insulated from each other. Since epitaxial growth of superconducting

\footnotetext{
* Corresponding author. Tel.: +90-232-750-75-10; fax: +90-232-75075-09.

E-mail address: ilbeyiavci@iyte.edu.tr (I. Avci).
}

YBCO layer is essential for the device fabrication, suitable buffer layers are usually needed between superconducting YBCO layers for circumventing the interdiffusion problem while keeping the epitaxy of the top layer [1-4].

Ion implantation has widely been used in the semiconductor technology in the last few decades for doping and modifying the semiconductor layers. In high temperature superconducting materials, ion implantation technique has been employed for the processing of oxide thin films suitable for the fabrication of superconducting devices [5]. The improvement in the current density and crystallinity of YBCO with $\mathrm{O}^{+}$ion implantation was studied by Non et al. [6], Lia et al. has studied the superconductivity of YBCO via $\mathrm{C}^{+}$implantation [7], patterning of $\mathrm{YBCO}$ thin films by $\mathrm{O}^{+}$ion implantation was studied by Kuhn et al. [8], the study of ion implantation and mass transport in YBCO were made by Kilner et al. [9]. In ion implantation, accelerated ions create point defects, which cause significant changes in superconducting properties of YBCO layers depending on the dose of the implanted ion and energy. In this work, we 


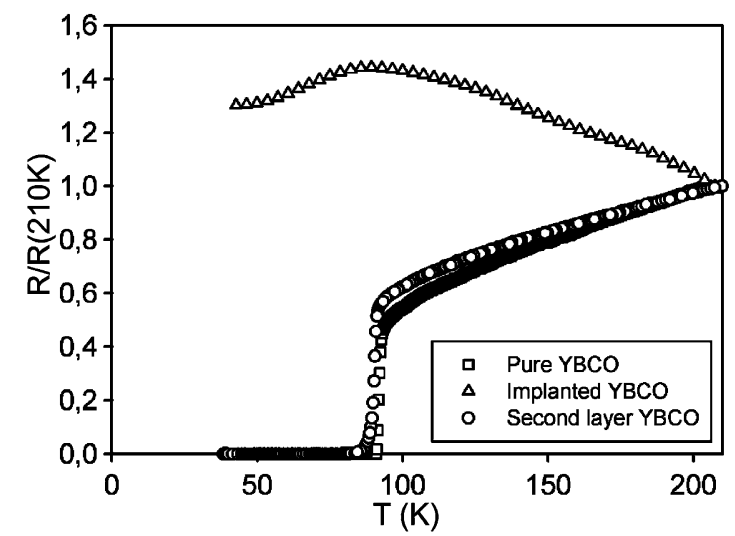

Fig. 1. Reduced resistance-temperature measurements of the pure, Si ion implanted - annealed and second layer YBCO samples.

have looked for obtaining Josephson junctions by making microbridges on YBCO multilayer structures which are interfaced across a Si-ion implanted top layer formed on the base layer over deposited onto $\mathrm{MgO}$-substrates, expecting to eliminate buffering with various materials. The results obtained were found satisfying our expectations, and encouraging to take further steps in making superconducting devices by employing this method.

\section{Experimental details}

The epitaxial $\mathrm{YBa}_{2} \mathrm{Cu}_{3} \mathrm{O}_{7}-\delta$ thin film was deposited 'in situ' by using DC inverted cylindrical magnetron sputtering (ICMS) technique on $(10 \times 10 \times 0.5 \mathrm{~mm})$-sized $(100)$ $\mathrm{SrTiO}_{3}$ substrate. This sputtering method is an effective technique for reproducibility of the good quality films [10]. The sputtering system was commercially purchased from Hitec-Materials having the high purity of YBCO target. The target-substrate distance was optimized at $35 \mathrm{~mm}$, substrate temperature was $830{ }^{\circ} \mathrm{C}$ and the plasma power was $40 \mathrm{~W}$. The film thickness was typically approximately $150 \mathrm{~nm}$ and the crystalline structure was fully $c$-axis oriented in accor-

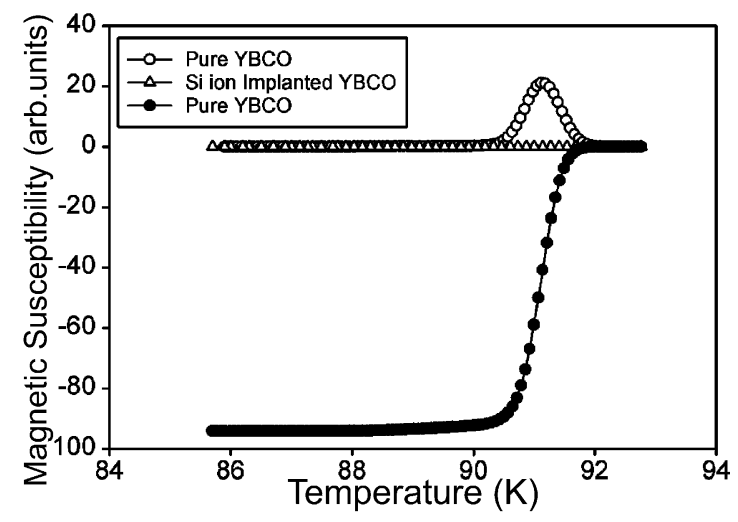

Fig. 2. AC magnetic susceptibility measurements of the pure and $\mathrm{Si}$ ion implanted - annealed YBCO samples.

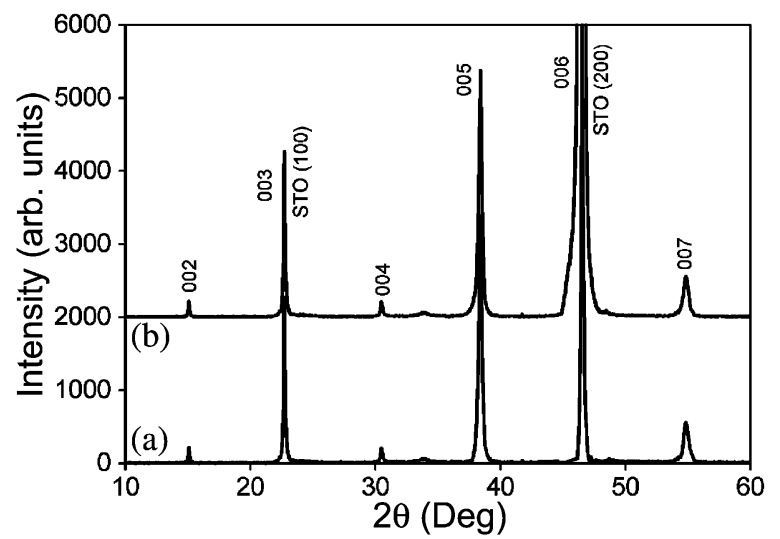

Fig. 3. XRD patterns of the samples, (a) pure YBCO and (b) Si ion implanted - annealed YBCO.

dance with a full width half maximum (FWHM) value of the rocking curve of $(005)$ peak $\leq 0.23^{\circ}$. The critical temperature $T_{\mathrm{c}}$ of the sample was approximately $91 \mathrm{~K}$ with very sharp transition and the resistively measured critical current density $J_{\mathrm{c}}$ was approximately $1.48 \times 10^{6} \mathrm{~A} / \mathrm{cm}^{2}$ at $77 \mathrm{~K}$ with zero magnetic field.

This film was implanted with an energy of $100 \mathrm{keV}$, $1 \times 10^{16} \mathrm{Si}$-ions $/ \mathrm{cm}^{2}$ at room temperature. Beam current density was approximately $0.3 \mu \mathrm{A} / \mathrm{cm}^{2}$. The ion distribution was estimated by the computer simulation program TRIM, which is realistic with respect to the experiment [11]. The result of TRIM calculation is shown in Fig. 4. Due to the low diffusion coefficient, $\mathrm{Si}$ distribution is expected to remain unchanged during the subsequent annealing and epitaxial growth processes.

The implanted sample was annealed in oxygen for 45 min starting from room temperature to the YBCO growth temperature of $830{ }^{\circ} \mathrm{C}$. The annealed sample was analyzed by measuring its resistance, AC magnetic susceptibility, Xray diffraction (XRD), scanning electron microscopy (SEM) and energy dispersive X-ray (EDX). The implanted and annealed sample was used for the growth of second layer YBCO film on top of the implanted area by ICMS on the

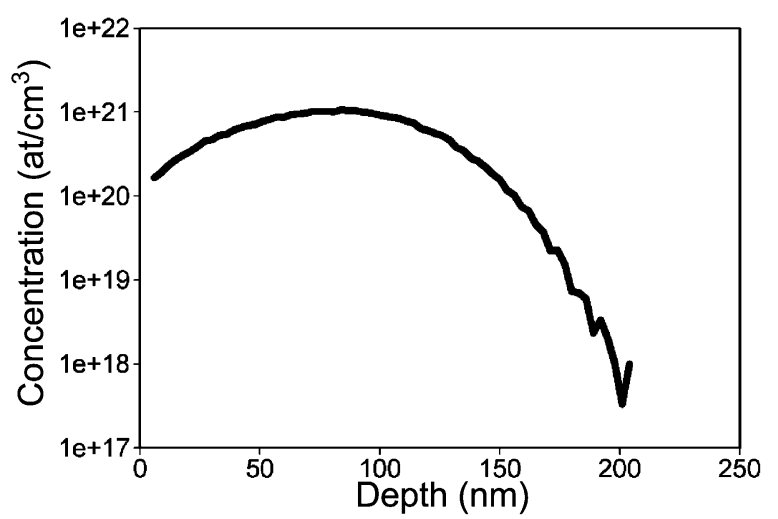

Fig. 4. TRIM profile of the Si ion implantation into the YBCO thin film. 


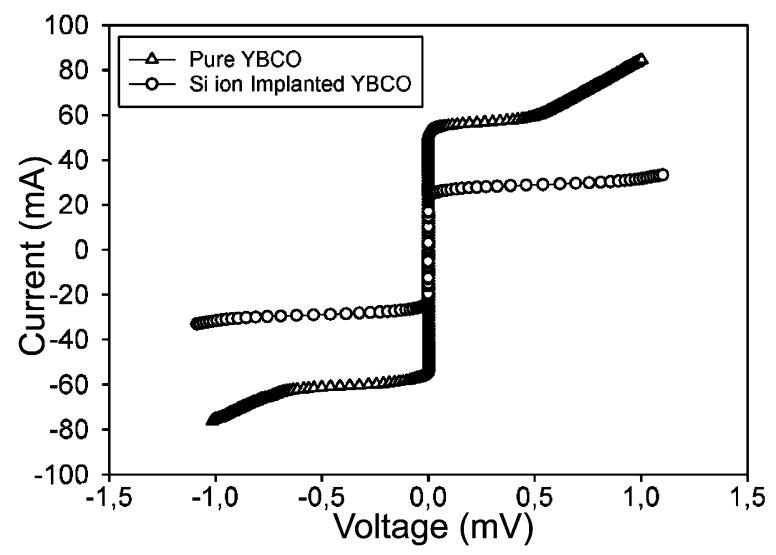

Fig. 5. $I-V$ measurements of the pure and second layer laser patterned YBCO microbridge at $77 \mathrm{~K}$.

same sputtering conditions with the first layer. The obtained YBCO multilayer film structure was patterned by 'laser writing technique' [12]. This technique was used to make a microbridge using a commercial solid state laser system which has computer controlled $X-Y$ translation stage. The film was patterned by focused laser beam to give a form approximately $20 \mu \mathrm{m}$ wide microbridge at the center of the sample. The critical current density of YBCO multilayerstructured microbridge was investigated by current-voltage measurement at $77 \mathrm{~K}$.

\section{Results and discussion}

In this study, we report on the Si ion implantation into the superconducting YBCO thin film and growth of second layer film on top of the implanted area. This study also reports the superconducting properties of laser patterned microbridge onto the second layer film. The second layer film and the microbridge patterned on that by laser writing technique, showed the superconducting properties similar to those of pure YBCO base layer with a reduced critical current density of approximately $42 \%$.

To investigate the superconducting properties of the sputtered YBCO film, electrical, magnetic, and structural measurements were performed by R-T, AC complex susceptibility, and XRD, respectively. The sample has showed very sharp transition at temperature approximately $91 \mathrm{~K}$, with a transition width of $\Delta T<0.5 \mathrm{~K}$ as shown in Fig. 1. Real $\left(\chi^{\prime}\right)$ and imaginary $\left(\chi^{\prime}\right)$ parts of the AC magnetic susceptibility response for an applied magnetic field are depicted in Fig. 2. The sharpness of the transitions seen in those figures indicates to the quality and the strong diamagnetic property of the film. XRD result of the pure YBCO film is shown in Fig. 3, curve a. The full width at half maximum (FWHM) value measured from the rocking curve on the YBCO (005) peak is $0.23^{\circ}$. This indicates a highly $c$-axis oriented film.

Prepared and analyzed film was implanted with an energy of $100 \mathrm{keV}, 1 \times 10^{16} \mathrm{Si}$-ions $/ \mathrm{cm}^{2}$ at room temperature without a mask and subsequently annealed in oxygen atmosphere for $45 \mathrm{~min}$ to recover the depleted oxygen and crystal defects in the structure. As can be seen from Fig. 4, the ion implantation created a defected layer with a thickness of $200 \mathrm{~nm}$. Some of these defects are expected to be repaired after the annealing process. To investigate the electrical and structural properties of the implanted YBCO film, R-T and XRD measurements were performed. Fig. 1 shows the resistance-temperature, (R-T), behavior of the implanted - annealed sample. It is seen that the resistance

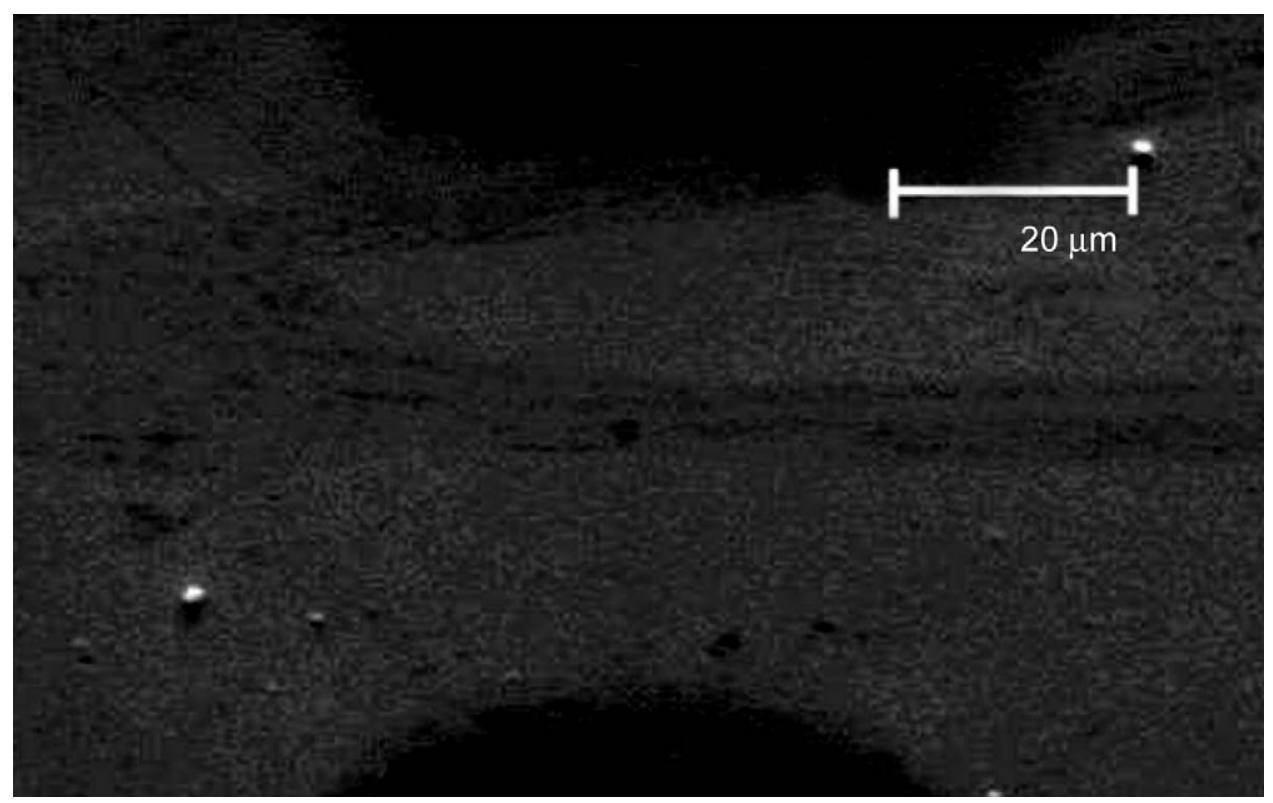

Fig. 6. SEM image of the laser patterned-second layer YBCO microbridge. 


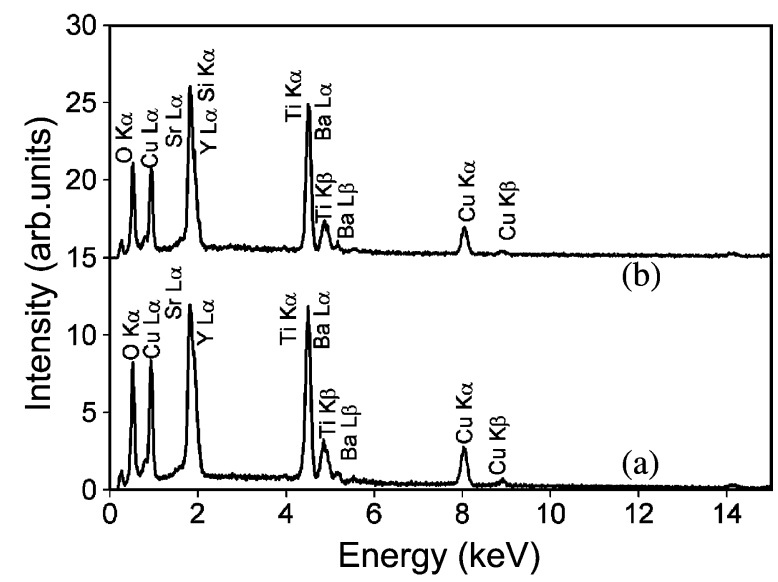

Fig. 7. EDX analysis of the samples, (a) pure YBCO and (b) $\mathrm{Si}$ ion implanted - annealed YBCO.

of the sample begins to decrease from $90 \mathrm{~K}$ down to $47 \mathrm{~K}$ and at lower temperatures below $47 \mathrm{~K}$. The resistance remains almost constant at the value of $530 \Omega$. This decrease in resistance at $90 \mathrm{~K}$ results from the un-implanted lower part of the YBCO film. Fig. 2 shows the nondiamagnetic behavior in accordance with the AC susceptibility measurement of the implanted - annealed sample. Fig. 3, curve $b$ shows the XRD patterns of the implanted annealed YBCO thin film. Fig. 3, curve $a$ and $b$ show that the crystal structure of $\mathrm{Si}$ ion implanted YBCO is similar to that of the pure sample. The FWHM value measured from the rocking curve on the YBCO (005) peak is $0.29^{\circ}$, which indicates the $c$-axis orientation is unchanged. This suggests that the Si ion implantation has no significant effect on the YBCO crystal structure.

After the annealing of the implanted sample, second layer YBCO thin film was sputtered directly on top of the implanted area. Fig. 1 shows the superconducting transition in resistance of the second layer YBCO film at the temperature approximately $90 \mathrm{~K}$ having small tail around $T_{\mathrm{c}} . I-V$ characteristic of the laser patterned microbridge on the second layer film shows the critical current density, $J_{\mathrm{c}}$, of $0.62 \times 10^{6} \mathrm{~A} / \mathrm{cm}^{2}$ at $77 \mathrm{~K}$ (Fig. 5, curve b). These $T_{\mathrm{c}}$ and $J_{\mathrm{c}}$ values of the second layer film and the microbridge show that the high- $T_{\mathrm{c}}$ superconducting multilayer YBCO thin film structure can be directly grown on top of the implanted area in good quality without using any buffer layer. Fig. 6 shows the SEM image of the laser written second layer YBCO microbridge where the edges of the geometry are sharp enough to calculate the width of the bridge from the center of the strip line ( $\approx 20 \mu \mathrm{m}$ ) and the surface of the second layer film is smooth. Fig. 7 shows the EDX analysis of the pure and Si-implanted YBCO films. The epitaxial growth of multilayer thin film structures as 'sandwich type' requires some modifications at the interfaces between the layers. A suitable buffer layer for this purpose has been commonly employed by the researchers. In $\mathrm{Si}$ ion implantation technique that we used, the second layer YBCO thin film has been directly grown on top of the implanted area of the first layer having similar superconducting properties with the pure YBCO. This technique, we believe, is an effective way for the fabrication of multilayer superconducting structures for making the building blocks of the superconducting electronic devices.

\section{Conclusion}

We have presented the fabrication of bilayer superconducting YBCO thin film by Si ion implantation and microbridge on this multilayer structure. The implanted film completely lost its superconductivity and diamagnetism while its crystalline structure was the same with that of the pure YBCO. This allows the growth of second layer superconducting YBCO film on top of the implanted area in good quality. This was supported by $T_{\mathrm{c}}$ of $90 \mathrm{~K}$, high- $J_{\mathrm{c}}$ approximately $10^{6} \mathrm{~A} / \mathrm{cm}^{2}$ at $77 \mathrm{~K}$, and highly $c$-axis orientation. In conclusion, $\mathrm{Si}$ ion implantation technique is an effective way to build epitaxial bilayer YBCO thin film structure. With the employment of the passivation of the Siimplanted layers for improving the interface properties and reducing the Si-diffusion in the layers, this technique could be extended into a multilayer process for the Josephson junction based electronic device production.

\section{Acknowledgements}

The authors would like to express their gratitude to specialists Gökhan Erdoğan and Duygu Oğuz for their valuable suggestions in the analysis of SEM, XRD and EDX at the Material Research Center at Izmir Institute of Technology.

\section{References}

[1] I. Belousov, P. Kus, S. Linzen, P. Seidel, Solid State Electron. 43 (1999) 1101.

[2] F. Kahlmann, A. Engelhardt, J. Schubert, W. Zander, Ch. Buchal, J. Hollkott, Nucl. Instrum. Meth. B 148 (1999) 803.

[3] P. Lee, K. Char, M.S. Colcough, G. Zaharchuk, Appl. Phys. Lett. 59 (1991) 3051

[4] M.J. Burns, K. Char, B.F. Cole, W.S. Ruby, S.A. Sachtjen, Appl. Phys. Lett. 62 (1993) 1435.

[5] J.R. LaGraff, G.Z. Pan, K.N. Tu, Physica C 338 (2000) 269.

[6] H.Y. Nan, X. Yao, M. Murakami, S. Tanaka, Physica C 282-287 (1997) 1043.

[7] X.T. Liu, H.X. Lu, X.H. Liu, Q.S. Chen, S.M. Pan, Physica C 282-287 (1997) 2547.

[8] M. Kuhn, B. Schey, R. Klarmann, W. Biegel, B. Stritzker, J. Eisenmenger, P. Leiderer, Physica C 294 (1998) 1.

[9] J.A. Kilner, Y. Li, Nucl. Instrum. Meth. B 139 (1998) 108.

[10] X.X. Xi, G. Linker, O. Meyer, E. Nold, B. Obst, F. Ratzel, R. Simithey, B. Strehlau, F. Weschenfelder, J. Geerk, Z. Phys. B 74 (1989) 13.

[11] J.F. Ziegler, SRIM, IBM Research, Yorktown Hights, NY, 1996.

[12] R. Sobolewski, W. Xiong, W. Kula, W.N. Maung, D.P. Butler, Supercond. Sci. Technol. 7 (1994) 300. 